\title{
Method for Direct Deconvolution of Heat Signals in Transient Adsorption Calorimetry
}

Christopher A. Wolcott ${ }^{\mathrm{a}}$, and Charles T. Campbell ${ }^{\mathrm{a}, \mathrm{b}, *}$

\author{
${ }^{a}$ University of Washington, Department of Chemical Engineering, Seattle, WA, USA \\ ${ }^{\mathrm{b}}$ University of Washington, Department of Chemistry, Seattle, WA 98195-1700, USA
}

*corresponding author, campbell@chem.washington.edu

\begin{abstract}
A method of heat signal analysis is presented for transient adsorption calorimetries including single crystal adsorption calorimetry (SCAC) which uses fast Fourier transforms (FFT) to determine the instrument response function and deconvolute the heat-versus-time signals. The method utilizes a heat signal generated by a laser pulse of known power-versus-time to extract the instrument response function for the calorimeter. The instrument response function is then used to extract the heat power signal from a molecular beam heat pulse of unknown intensity. This method allows for the extraction of the total heat deposited by the molecular beam pulse without any kinetic modeling even in the event of complex reaction dynamics. This method is compared to previous methods used to analyze SCAC data using example data from the two-step dissociative adsorption of methyl iodide on $\mathrm{Pt}(111)$. It is found to be equally accurate for extracting total heats and simpler to perform than the previous methods.
\end{abstract}

Key Words: SCAC, Single Crystal Adsorption Calorimetry, Transient Adsorption Microcalorimetry

\section{Introduction}

The measurement of reaction enthalpies for elementary steps occurring on solid surfaces is crucial for fundamental understanding in heterogeneous catalysis and many other technologies. Few techniques exist to measure these quantities directly, especially on single crystal surfaces which allow for the adsorbed species to be fully characterized using a variety of surface science 
techniques and computationally modelled using state-of-the-art density function theory (DFT) with periodic boundary conditions. Typical techniques used to extract reaction enthalpies for surface processes are indirect, such as temperature programmed desorption (TPD) and equilibrium adsorption experiments (typically performed in either isobaric or isothermal conditions). These techniques require that a process be reversible to extract thermodynamic information such as the heat of adsorption for a particular surface species ${ }^{1}$. Since many surface processes of interest, especially in heterogeneous catalysis are irreversible processes such as dissociative or reactive adsorption, these techniques cannot be used to probe many important species. Though some qualitative information about the stability of molecular fragments can be gained through very clever application of these classic techniques such as the work of Friend et al. (add cite when published) looking at competitive adsorption of different species on a surface, quantitative information remains largely unattainable. Common surface processes such as dissociative adsorption or other surface reactions require a different technique in order to extract quantitative thermodynamic information about adsorbed species.

Single crystal adsorption calorimetry was developed to study the energetics of adsorption on single crystal surfaces directly ${ }^{2}$. It does not require that a process be reversible, nor that a kinetic model be developed to extract the heats of adsorption in most cases. A tremendously wide range of adsorbates have been studied using this technique, from simple gases ${ }^{3}$ like CO, $\mathrm{NO}, \mathrm{O}_{2}$, and $\mathrm{C}_{2} \mathrm{H}_{4}$, to larger, low vapor pressure molecules like benzene ${ }^{4}$, napthalene ${ }^{5}$, cyclohexene $^{6}$, $_{\text {ater }}{ }^{7-9}$, methanol $^{10}$, formic acid ${ }^{11}$, methyl iodide ${ }^{12,13}$, t-butyl iodide ${ }^{14}$, and diiodomethane ${ }^{15}$, and even to metals like $\mathrm{Ca}^{16}, \mathrm{Li}^{16,17}, \mathrm{~Pb}^{18-20}, \mathrm{Ag}^{21,22}$, and $\mathrm{Cu}^{23}$. The same general type of technique that works on single crystals and is known as SCAC also works on many other types of planar surfaces, and has recently been applied to $\mathrm{CO}$ adsorption on oxide supported metal nanoparticles ${ }^{24}$, metal adsorption on spin-coated polymers ${ }^{25,26}$ and even to electrochemical systems ${ }^{27,28}$ with a solid-liquid interface. For the purposes of this present paper, we describe these more generally as "transient adsorption microcalorimetry", and the signal analysis techniques we describe below apply to all such methods. In many of these systems, the heat that is generated upon adsorption is transferred nearly instantaneously to the calorimeter's heat detector, allowing a simple calibration to a known heat signal to be used to extract the adsorption heat of the unknown process. In these cases, simply scaling the calibration signal until it matches the unknown signal exactly lets one determine the amount of energy in the unknown 
signal. We address here what happens when the heat deposition instead is not instantaneous, and occurs with some time delay on the same millisecond time scale as the heat measurement, due to slower surface reaction kinetics.

This phenomena of a non-instantaneous or "slow" heat deposition was first reported by Lew et al. ${ }^{7}$, who observed that the calorimeter signal seen for the adsorption of $\mathrm{D}_{2} \mathrm{O}$ on a $\mathrm{Pt}(111)$ surface did not possess the same normalized lineshape as the calibration signal generated with a laser pulse (which deposits its heat to the sample's surface instantaneously). The heat signal pulse appeared to be "broadened" relative to the laser signal, possessing a larger full width at half max (FWHM). The mismatch was very slight, with only a 6\% mismatch between the FWHM of the measured heat signal and the laser calibration signal. By convoluting the measured laser calibration signal with an exponential decay function with a time constant of $\sim 30 \mathrm{~ms}$, they were able to produce an identical signal to the heat signal measured, suggesting the heat of adsorption was produced by a single slow process which had a characteristic time constant equal to $\sim 30 \mathrm{~ms}$. This slow heat process was attributed to adsorption and relaxation of the adsorbed $\mathrm{D}_{2} \mathrm{O}$ molecules into their most stable packing arrangement on the $\mathrm{Pt}(111)$ surface.

This slow heat deposition phenomenon was observed again for the adsorption of methyl iodide on a $\mathrm{Pt}(111)$ surface $^{12}$. In this instance the heat of adsorption could not be fit using the convolution method of Lew et al., suggesting that the process was more complex than a single slow process. A new method was developed by Silbaugh et al. ${ }^{29}$, which modelled the adsorption process as a single instantaneous "fast" step followed by a slower step modelled by convoluting the laser signal with an exponential decay function as was done in the method of Lew et al.. The sum of the signals from these "fast" and "slow" steps could then reproduce the measured signal. This method had three variables to fit, the heat of the fast step, the heat of the slow step, and the time constant for the slow step time decay function. When appropriately fit, this method produced a unique solution which yielded not only the heats of both the initial fast step and second slow step, but also for the first-order rate constant for the slow reaction (i.e., the inverse of the time constant obtained from the fit). This powerful method, while labor intensive as it requires fitting three parameters for each pulse's lineshape (or an average lineshape from a small number of pulses), yields a wealth of information on adsorption energetics and dynamics. In addition to being used for methyl iodide adsorption on $\mathrm{Pt}(111)$ to yield information about the 
heat of adsorption of $\mathrm{CH}_{3} \mathrm{I}, \mathrm{CH}_{3}$, and the dynamics of the reaction $\mathrm{CH}_{3} \mathrm{I}_{\mathrm{ad}} \rightarrow \mathrm{CH}_{3, \mathrm{ad}}+\mathrm{I}_{\mathrm{ad}}$, this method has also been used to study the adsorption of formic acid on $\operatorname{Pt}(111)^{11}$, yielding information about the energetics of molecular formic acid, monodentate and bidentate formate, and kinetic information about the reactions $\mathrm{HCOOH}_{\mathrm{ad}}+\mathrm{O}_{\mathrm{ad}} \rightarrow \mathrm{HCOO}_{\mathrm{ad} \text {,mono }}+\mathrm{OH}_{\mathrm{ad}}$ and $\mathrm{HCOO}_{\mathrm{ad}, \mathrm{mono}} \rightarrow \mathrm{HCHOO}_{\mathrm{ad}, \mathrm{bi}}$. Both of these methods present valuable and highly functional techniques for analyzing simple adsorption-relaxation and adsorption-reaction processes. Through the development of a mechanistic and kinetic model, they allow the extraction of both thermodynamic and kinetic information in whatever system they are applied. They each however, have their limitations. The key limitation is that they only work for the precise processes they model, i.e. a single slow step or a combination of a single fast step followed by a single slow step. While in theory the method of Silbaugh et al. could be expanded to include any number of fast and slow steps occurring either in series or parallel, this rapidly becomes infeasible as the number of parameters which are necessary to fit increases, making it more labor intensive and at the same time harder to show the uniqueness of a solution. As a result a new method is needed to analyze any reaction with more complex surface reaction dynamics than those modelled by Lew and Silbaugh.

This paper presents a more general method for analysis of transient adsorption calorimetry data which requires no kinetic modelling or parameter fitting even in the event of signal broadening. This new method utilizes FFTs to extract heat data directly from the calorimeter signal without any scaling or kinetic parameters. This method successfully yields the total heat deposited on the surface regardless of any signal broadening. This method possesses universal applicability and a complete absence of fitting parameters or requirements for pulse averaging give it many distinct advantages. This technique is demonstrated here by applying it to study the heat of adsorption of diiodomethane on $\operatorname{Pt}(111)$, whose adsorption, reaction, and diffusion dynamics were sufficiently complex that they could not be modelled using the method of Silbaugh et al.. A more extensive presentation of the results of this application is presented elsewhere ${ }^{15}$.

\section{Signal Generation and Pulse Shape}

We describe here the technique is association with SCAC, although the same principles could be applied to any transient adsorption calorimetry. For the purposes of clarity, a brief 
explanation of the SCAC technique itself will be given first. The explanation given here is for ultrahigh vacuum (UHV) gas / solid interactions, which is the most common form of SCAC. For full details of this version of SCAC, see Ajo et al.. ${ }^{30}$ For details of the electrochemical version of SCAC, see Schuster et al.. ${ }^{31}$

SCAC is typically performed in a stainless steel UHV chamber (base pressure $<2 \times 10^{-10}$ mbar). Other surface science techniques such as Auger electron spectroscopy (AES), low energy ion scattering (LEIS), low-energy electron diffraction (LEED), and x-ray photoelectron spectroscopy (XPS) are used to verify sample cleanliness and order. A very thin (typically $1 \mu \mathrm{m})$ single crystal is used as the sample, to minimize thermal mass while maintaining structural stability. A heat detector, typically a pyroelectric polymer ribbon made of $\beta$-polyvinylidyne fluoride $(\beta-\mathrm{PVDF})$, is driven into gentle contact with the back of the sample, and is then used to measure minute temperature changes in the sample upon heat adsorption, which are recorded as a transient voltage signal after preamplification and further amplification and electronic signal filtering. This gives rise to the observed instrument response lineshape, as discussed previously ${ }^{29,32,33}$. The heat detector is calibrated using a laser pulse whose intensity is measured using a photodiode. Molecules are dosed using a molecular beam and the sticking fraction of molecules is measured using the King-Wells method ${ }^{34}$. The duration of a pulse, either laser or molecular, is controlled by chopping the beam with the same mechanical chopper. The flux of molecules is measured using a liquid nitrogen cooled quartz crystal microbalance (QCM). Each pulse of molecules or laser is typically around $100 \mathrm{~ms}$ long. The output from the detector is recorded as a voltage signal as a function of time shown, such as in the top of Fig. 1.

If the heat deposition from the molecular beam pulse occurs rapidly $(<10 \mathrm{~ms})$ the resulting detector signal will have the identical shape as the laser pulse (which also deposits heat extremely rapidly). By simply scaling the molecular beam pulse to match the magnitude of the laser pulse, whose intensity is known, the amount of heat deposited by the molecular beam pulse can be found.

If the heat deposition is on the same timescale as the pulse duration $(\sim 10 \mathrm{~ms}-1000 \mathrm{~ms})$ then the molecular beam pulse appears broadened, as shown in Fig. 1. This figure shows a superposition of the laser calibration response and the heat deposition of $\mathrm{CH}_{2} \mathrm{I}_{2}$ on $\mathrm{Pt}(111)$ at $125 \mathrm{~K}$. In this case, a more complex analysis is necessary. The approach depends on what the 
expected reaction dynamics are. If the reaction is simple, consisting of two or fewer steps, then the method of Silbaugh et al. ${ }^{29}$ can be used. An example of a two-step process where this occurs is shown in Eq. 1.

$$
\mathrm{CH}_{3} \mathrm{I}_{\mathrm{g}} \stackrel{\text { fast }}{\longrightarrow} \mathrm{CH}_{3} \mathrm{I}_{\mathrm{ad}} \stackrel{\text { slow }}{\longrightarrow} \mathrm{CH}_{3, \mathrm{ad}}+\mathrm{I}_{\text {ad }}
$$

A model is generated consisting of the sum of scaled laser pulse and another scaled laser pulse which has been convoluted with an exponential decay function shown in Eq. 2 .

$$
D=1 / \tau e^{-t / \tau}
$$

The scaling factors of each of the pulses and the time constant in the exponential decay function are varied until the sum of the two pulses matches the measured pulse. The method of Lew et al. discussed in the Introduction can be seen as identical to the method of Silbaugh et al. if the instantaneous heat is zero. If there is a heat deposition process which occurs with a time constant slower than the time between pulses, it is not measurable using SCAC and will appear as if it is not occurring, since it contributes only to an increase in the background. In practice, even processes that occur with a time constant longer than $1 / 5$ of this (i.e., $>1 \mathrm{~s}$ ) are difficult to see about the background noise, since such extreme broadening decreases pulse amplitude too much.

If the adsorption process has more than one step with a rate constant on the same order as the pulse duration, then in theory it could be fit using the method of Silbaugh et al.. This approach of convoluting multiple contributions to try to fit the signal rapidly becomes unfeasible, however, as more variables are added along with the possibility of each additional step occurring either in series or in parallel with other steps. In addition to being extremely labor intensive, one should be skeptical of the uniqueness of any solution found when very many variables are needed to fit the data.

This scenario of multiple slow steps was encountered when studying the energetics of diiodomethane on $\operatorname{Pt}(111)$. The dissociation of diiodomethane (Eq. 3) on $\operatorname{Pt}(111)$ to produce $\mathrm{CH}_{\mathrm{ad}}, 2 \mathrm{I}_{\mathrm{ad}}$, and $\mathrm{H}_{\mathrm{ad}}$ is expected at temperatures as low as $130 \mathrm{~K}^{35}$.

$$
\text { (1) } \mathrm{CH}_{2} \mathrm{I}_{2, g} \stackrel{\text { dissoc.+diff. }}{\longrightarrow} \mathrm{CH}_{2, a d}+2 I_{a d} \stackrel{\text { dissoc.+diff. }}{\longrightarrow} \mathrm{CH}_{a d}+2 I_{a d}+H_{a d} \text {. }
$$


This process involved the breaking of two $\mathrm{C}-\mathrm{I}$ bonds, one $\mathrm{C}-\mathrm{H}$ bond, as well as surface diffusion of strongly bound surface species such as $\mathrm{I}_{\mathrm{ad}}$ and $\mathrm{CH}_{\mathrm{x}, \text { ad }}$. If any two or more of these processes are slow, then the data cannot be analyzed using the method of Silbaugh et al.. Attempts were made using the two step method Silbaugh et al. to fit the data, but the complete reaction dynamics could not be captured (as evidenced by a poor fit of the models to the data). Attempts were made to modify the method of Silbaugh to include a third step either in series or in parallel and even this did not seem to capture the complete reaction dynamics (again evidenced by a poor fit). To extract meaningful heats out of the signals, a different approach was necessary.

\section{Direct heat extraction using FFT}

The shape of the detector response signal, $\mathrm{S}(\mathrm{t})$,results from the convolution of a heat input $(\mathrm{I}(\mathrm{t}))$ with an instrument response function $\left(\mathrm{R}\left(\mathrm{t}-\mathrm{t}^{\prime}\right)\right)$ :

$$
S(t)=(I * R)(t)=\int_{-\infty}^{\infty} I\left(t^{\prime}\right) R\left(t-t^{\prime}\right) d t^{\prime}
$$

For the heat detector used in this paper the functions, $\mathrm{S}(\mathrm{t}), \mathrm{I}(\mathrm{t})$, and $\mathrm{R}(\mathrm{t})$ are shown graphically in Fig. 2. If the heat input is known for any given measured detector response signal, it is possible to use deconvolution via FFT to extract the instrument response function from the measured detector response signal. This is done by first taking the a discrete Fourier transform of the measured signal, $\boldsymbol{F}\{\mathrm{S}(\mathrm{t})\}$, which allows the convolution to be split into the product of two separate Fourier transforms, as a direct mathematical consequence of Eq. (4) above:

$$
\boldsymbol{F}\{S(t)\}=\boldsymbol{F}\{(I * R)(t)\}=\boldsymbol{F}\{I(t)\} \boldsymbol{F}\{R(t)\}
$$

Equation (5) is only true when the detector response is linear. One should always test the calorimeter response during calibration with different laser powers to ensure that the signal magnitude scales linearly with laser power and the lineshape is independent of power, as described previously ${ }^{32,33}$. Simple mathematical rearrangements, followed by an inverse Fourier Transform, then provides a direct solution for the instrument response function via:

$$
R(t)=\boldsymbol{F}^{-1}\{\boldsymbol{F}\{S(t)\} / \boldsymbol{F}\{(I(t)\}\}(t),
$$

where $\boldsymbol{F}^{-1}\{\boldsymbol{X}\}$ represents the inverse Fourier Transform of $\boldsymbol{X}$. 
Similarly if the instrument response function is known for any given measured detector response signal but the heat input is unknown, the heat input can be found using an identical method only solving for $I(t)$ instead of $R(t)$ :

$$
I(t)=\boldsymbol{F}^{-\mathbf{1}}\{\boldsymbol{F}\{S(t)\} / \boldsymbol{F}\{(R(t)\}\},
$$

During laser calibration of the calorimeter, the magnitude of the heat input from the laser pulses is measured with a photodiode and properly scaled to correct for imperfect transmittance through a window on the UHV chamber (which separates the photodiode from the sample). The pulse width is of both the laser and the molecular beam is set by adjusting the speed of the chopper in the molecular beam line. The laser pulse lineshape is trapezoidal and can be calculated exactly from the known geometry of the chopper blade. However, it is closely approximated by a square wave as shown in Fig. 2. This square wave "model" of the laser heat input was then used to extract the instrument response function from the detector response to the laser pulse (as shown in Fig. 2) using the mathematical procedure described above. We also used the true trapezoidal lineshape of the laser pulse, and found this to produce results that were indistinguishable from the approximate square-wave lineshape, so we use this simpler squarewave model in the results below.

All calculations and FFTs were performed in Wolfram Mathematica 9.0 using its built-in functions. Once the instrument response function had been found, the same analysis can be done using the detector response signal for the molecular beam pulses, only solving for the heat input instead. An example result showing one heat input for a pulse of $\mathrm{CH}_{2} \mathrm{I}_{2}$ adsorbed on $\mathrm{Pt}(111)$ at $125 \mathrm{~K}$ is shown in Fig. 3. Superimposed on Fig. 3 is the laser power model square wave used to extract the instrument response function from the average of 50 laser pulse signals, and an individual laser pulse heat input which was deconvoluted using that instrument response function. Immediately, it is clear that the heat input of the molecular beam pulse is broadened to longer times compared to the laser pulse heat input. Additionally it can be seen in Fig. 3 that there is a periodic baseline noise in the deconvoluted signal, which is an unavoidable mathematical artefact of the deconvolution procedure. The deconvoluted heat signal presented in Fig. 3 has already been low-pass filtered and further filtering to attempt to remove the periodic noise which remains resulted in a change in the shape and magnitude of the signal. No 
apodization functions were used in this FFT, although it is possible they would clean the signal even more.

It is obvious that integrating the heat input obtained in Fig. 3 would yield the adsorption energy for that pulse. Integrating across the entire five-second pulse period would be the ideal integration window for this. However, the baseline noise present in the deconvoluted signal contributes to noise in this time-integrated heat, and the magnitude of that noise contribution grows with increasing integration time, as shown below. The best integration window depends on the time duration of the actual heat input, which varies with the reaction being studied and surface temperature. In general, heat deposition for first-order processes shows as an exponential time decay with some characteristic time constant, $\tau$. The limits of the integration window can then be understood in terms of the fraction of heat captured at specific times after the pulse center. By integrating out to $1 \tau, 63 \%$ of the heat is captured, $2 \tau$ yields $86 \%$, and $3 \tau$ yields $95 \%$, and so on, increasing as the integral of Eqn. 2. Alternatively, for any fixed time window, we can calculate the largest $\tau$ for which we capture $95 \%$ of the heat. In our example we chose a start time $200 \mathrm{~ms}$ before the middle of the molecular beam pulse (which is $102 \mathrm{~ms}$ long) and a stop time $\sim 500 \mathrm{~ms}$ after that. This means we miss any heat deposited slower than $\sim 500 \mathrm{~ms}$, and means we get $>95 \%$ of the heat for any reactions that happen with $\tau<\sim 170 \mathrm{~ms}$. Further discussion of the method for intelligent selection of the integration window is discussed below with numerical examples. By measuring the amount of molecules sticking in the pulse using the same methods typically used in SCAC, ${ }^{30}$ the heat of adsorption in kJ per mole adsorbed can be found from the time-integrated heat for each pulse.

Since the limited data range used for the Fourier transform deconvolution introduces periodic oscillations in the deconvoluted heat input, one should also fine-tune the integration start- and stop-times to fall halfway between the minima and maxima of these noise oscillations to avoid systematic error. It is easy to identify the periodicity and time locations of these minima and maxima by averaging multiple curves like Fig. 3. To assess the significance of this potential source of error, we stepped through the period of oscillation in $1 / 4$ increments on both ends of the integration window. The resulting heat vs. coverage plots are shown in Fig. 4. These heats are averaged across four sets of data collected upon the adsorption of $\mathrm{CH}_{2} \mathrm{I}_{2}$ on $\operatorname{Pt}(111)$ at $125 \mathrm{~K}$. The result shows that there is no systematic offset at almost all coverages produced by changing 
the integration windows in this way (Fig. 4a), showing that the periodic noise does not contribute significantly to the averaged or integral heat of adsorption. However, there is a systematic increase in the heats at very low coverage when the end point of integration is increased (Fig. 4b). These low-coverage pulses were the most broadened by contributions from the slow process, and this systematic increase is due to capturing more and more of this slowly deposited heat. This result highlights the problem with picking a window that is too short, and thus not capturing all of the heat input when a significant part of it occurs in a reaction step with a long time constant, as it does at very low coverages here.

Several different end points for integration were tested using the same raw sets of data as in Fig. 4 and are shown in Fig. 5. It can be seen that although the noise present in the data increases with increasing the size of the window, integrating beyond $1000 \mathrm{~ms}$ no longer changes the magnitude of the low-coverage heats as it did up to $750 \mathrm{~ms}$ (Fig. 4). By inspection of the raw pulse shapes, we found that the largest noise spikes in Fig. 5 all come from a few isolated spikes in the baseline signal which are picked up at large integration times but not at short times.

Figure 6 shows the pulse-to-pulse standard deviation of the heat in the coverage range 0.2-0.4 ML in Fig. 5 (where the heat is relatively constant) plotted versus the width of the integration window. As seen, the noise increases nearly proportional to the width of the integration window. Thus, to minimize noise, one should choose the shortest window that captures all of the heat, which unfortunately gets longer the slower the reaction rate for the heat deposition reactions being studied.

Returning to Fig. 4 in light of Fig. 6, it can be seen that the additional noise introduced by increasing the integration window from 300 to $400 \mathrm{~ms}$ there increased the standard deviation only by about $\sim 10 \mathrm{~kJ} / \mathrm{mol}$, a very small amount compared to the magnitude of the heat signals measured.

Striking the right balance between collecting as much slow heat deposition as possible in the integration window and minimizing the noise in the data is important in the use of this method. The appropriate selection of the end points of integration is clearly very important, and it must be carefully selected when using this technique. Since it depends on the time duration of 
heat input, which varies with the reaction kinetics, it will depend sensitively on both the reaction being studied and surface temperature.

\section{Comparison of Results to Method of Silbaugh}

To showcase the functionality of this new method, we reanalyzed a set of raw data which had been previously analyzed using the method of Silbaugh et al. ${ }^{12,29}$. The experiment consists of the measured heat of dissociative adsorption for $\mathrm{CH}_{3} \mathrm{I}$ on $\mathrm{Pt}(111)$ at $270 \mathrm{~K}$. A plot showing both the method of Silbaugh and the method of this paper are shown in Fig. 7. The heat plotted here is the total heat from Silbaugh, which is the sum of the slow and fast step heats from Eq. 1. In the method of Silbaugh, sets of four pulses were averaged in order to produce clean enough data to fit with that method. This averaging of multiple pulses was not necessary for the method of this paper, which can extract heat for every pulse individually. The two methods can be seen to yield nearly identical heat-versus-coverage plots when both are fitted to straight lines, as shown. Each point from Silbaugh's method should nearly equal the average of the four adjacent points around it using the method of this paper. This is indeed found to be the case, with an average difference of $6.5 \pm 11.8 \mathrm{~kJ} / \mathrm{mol}$ (95\% confidence) between the two sets of data when compared in that way. This can be compared to the $1.7 \mathrm{~kJ} / \mathrm{mol}$ pulse-to-pulse standard deviation of this particular SCAC instrument at similar conditions but when the data pulses show no broadening and can be quantified by simply comparing pulse amplitude to the laser calibration ${ }^{36}$.

One advantage of this FFT technique over the method of Silbaugh can be readily seen in Fig. 7 by the point at $\sim 0.009$ ML coverage, highlighted with a green arrow. Because the method of Silbaugh required the averaging of four pulses to get a clean enough lineshape to model, outlying points can be incorporated into that average without the knowledge of the person analyzing the data. That is not the case with this new method, and that highlighted point can easily be identified as an outlier, as shown here. Upon looking through the raw pulse lineshape for this pulse, it was found that this point was the result of a baseline noise spike.

The method of Silbaugh et al. ${ }^{29}$ also allows for the extraction of kinetic parameters as well as thermodynamic heats of adsorption. The details of this methodology are discussed briefly in the Introduction above, and in detail in that paper. Two attempts were made to extract kinetic information in the same vein as the method of Silbaugh et al but instead using the FFT 
deconvoluted heat pulse lineshape. The first approach was to simply fit the tail of the deconvoluted heat pulse with an exponential of the form of Eq. 2. Attempting to fit the same data of Silbaugh et al. but instead using this method resulted in fit parameters that were similar in magnitude to those reported by Silbaugh. However, it was found that even minor variations in the starting point of the fit would results in changes in the time constant by $50 \%$ or more. A method for properly determining the correct starting point could not be found, due to the small magnitude of the slow heat signal compared to the noise present in the deconvoluted signal.

To demonstrate that kinetic information can be obtained in principle by this FFT method when the signal / noise ratio is good enough, we simulated a nearly noiseless heat signal lineshape that would be expected in the absence of noise due to the heat of adsorption followed by dissociation for $\mathrm{CH}_{3} \mathrm{I}$ on $\mathrm{Pt}(111)$, as shown in Fig. 8. At the conditions modelled here, Silbaugh et al. ${ }^{29}$ showed that $\mathrm{CH}_{3}$ I first adsorbs molecularly with a heat of $184 \mathrm{~kJ} / \mathrm{mol}$ and then dissociates via a first-order reaction with time constant $\tau=170 \mathrm{~ms}$ and heat of $64 \mathrm{~kJ} / \mathrm{mol}$ Curve (a) at the bottom shows the reaction heat input to the sample versus time for this two-step process. The curve at the top shows the signal waveform simulated for this same heat input model generated using the simulation method of Silbaugh et al. ${ }^{29}$, which sums heat-scaled waveforms for its two steps. This simulation uses the nearly noiseless measured response to laser heating (averaged over many pulses) for the first, fast step and its convolution with firstorder kinetics for the slow step. Curve (b) at the bottom shows the heat input versus time obtained by deconvolution of this nearly noiseless signal to remove the instrument response function using the FFT method of this paper. As seen, curves (a) and (b) agree very closely, except for the damped periodic noise in (b). It is clear that one could model this red curve with the sum of the blue and green curves to extract values for the two separate heat contributions and the time constant for the exponentially decaying tail, although somewhat different values would be obtained due to the small amount of noise present even in this heat-versus-time curve.

\section{Conclusions}

A universal method is demonstrated to analyze transient adsorption calorimetry data regardless of reaction dynamics on the surface. Traditional methods ${ }^{7,29,30}$ require that the heat of adsorption consist of either a single fast step, a single slow step step, or a combination of a single fast and a single slow step in order to extract thermodynamic information. In the event of the 
presence of a slow step, these other methods require fitting the measured heat data with a mechanistic and kinetic model to obtain any heat information, something this new universal method does not require. In addition to being less labor intensive than these other methods, this new technique allows for the extraction of the total adsorbed heat from even extremely complex adsorption-reaction-diffusion-relaxation systems.

This new data analysis method has been applied to the study of the adsorption of diiodomethane on $\mathrm{Pt}(111)$, providing coverage dependent heats of adsorption for this system at temperatures ranging from $100 \mathrm{~K}$ to $210 \mathrm{~K}^{15}$. The complex, many-step dynamics of this system made it impossible to analyze using any of the previous methods, showcasing the unique power of this new method. The method has also been used to reanalyze and compare with data from Silbaugh et al. ${ }^{29}$, showing that both of the methods yield the same total heat of adsorption for a process consisting of a single fast and a single slow step.

\section{Author Information}

\section{Corresponding Author}

*E-mail: campbell@ chem.washington.edu.

\section{Notes}

The authors declare no competing financial interest.

\section{Acknowledgements}

The authors acknowledge support for this work by the US Department of Energy Office of Basic Energy Sciences (DOE-OBES) Materials Genome Initiative (MGI) by a subcontract through the Stanford University (Principal Investigator: Jens K. Norskov).

\section{References}

1. Ertl, G., Surface Science. Chemphyschem 2009, 10, 22-22.

2. King, D. A., Adsorption Calorimeter on Single-Crystal Surfaces - a new Technique. Physica Scripta 1993, T49B, 560-562.

3. Brown, W. A.; Kose, R.; King, D. A., Femtomole adsorption calorimetry on singlecrystal surfaces. Chemical Reviews 1998, 98, 797-831.

4. Ihm, H.; Ajo, H. M.; Gottfried, J. M.; Bera, P.; Campbell, C. T., Calorimetric measurement of the heat of adsorption of benzene on $\mathrm{Pt}(111)$. Journal of Physical Chemistry B 2004, 108, 14627-14633. 
5. Gottfried, J. M.; Vestergaard, E. K.; Bera, P.; Campbell, C. T., Heat of adsorption of naphthalene on $\mathrm{Pt}(111)$ measured by adsorption calorimetry. Journal of Physical Chemistry B 2006, 110, 17539-17545.

6. Lytken, O.; Lew, W.; Harris, J. J. W.; Vestergaard, E. K.; Gottfried, J. M.; Campbell, C. T., Energetics of cyclohexene adsorption and reaction on $\mathrm{Pt}(111)$ by low-temperature microcalorimetry. Journal of the American Chemical Society 2008, 130, 10247-10257.

7. Lew, W. D.; Crowe, M. C.; Karp, E.; Campbell, C. T., Energy of Molecularly Adsorbed Water on Clean $\mathrm{Pt}(111)$ and $\mathrm{Pt}(111)$ with Coadsorbed Oxygen by Calorimetry. Journal of Physical Chemistry C 2011, 115, 9164-9170.

8. Lew, W.; Crowe, M. C.; Campbell, C. T.; Carrasco, J.; Michaelides, A., The Energy of Hydroxyl Coadsorbed with Water on Pt(111). Journal of Physical Chemistry C 2011, 115, 23008-23012.

9. Lew, W.; Crowe, M. C.; Karp, E.; Lytken, O.; Farmer, J. A.; Arnadottir, L.; Schoenbaum, C.; Campbell, C. T., The Energy of Adsorbed Hydroxyl on Pt(111) by Microcalorimetry. Journal of Physical Chemistry C 2011, 115, 11586-11594.

10. Karp, E. M.; Silbaugh, T. L.; Crowe, M. C.; Campbell, C. T., Energetics of Adsorbed Methanol and Methoxy on Pt(111) by Microcalorimetry. Journal of the American Chemical Society 2012, 134, 20388-20395.

11. Silbaugh, T. L.; Karp, E. M.; Campbell, C. T., Energetics of Formic Acid Conversion to Adsorbed Formates on $\operatorname{Pt}(111)$ by Transient Calorimetry. Journal of the American Chemical Society 2014, 136, 3964-3971.

12. Karp, E. M.; Silbaugh, T. L.; Campbell, C. T., Energetics of Adsorbed CH3 and CH on $\mathrm{Pt}(111)$ by Calorimetry: Dissociative Adsorption of CH3I. Journal of Physical Chemistry C 2013, 117, 6325-6336.

13. Karp, E. M.; Silbaugh, T. L.; Campbell, C. T., Energetics of Adsorbed CH3 on Pt(111) by Calorimetry. Journal of the American Chemical Society 2013, 135, 5208-5211.

14. Silbaugh, T. L.; Giorgi, J. B.; Xu, Y.; Tillekaratne, A.; Zaera, F.; Campbell, C. T., Adsorption Energy of tert-Butyl on $\mathrm{Pt}(111)$ by Dissociation of tert-Butyl Iodide: Calorimetry and DFT. Journal of Physical Chemistry C 2014, 118, 427-438.

15. Wolcott, C. A.; Green, I. X.; Silbaugh, T. L.; Xu, Y.; Campbell, C. T., Energetics of Adsorbed $\mathrm{CH}_{2}$ and $\mathrm{CH}$ on $\mathrm{Pt}(111)$ by Calorimetry: The Dissociative Adsorption of Diiodomethane. The Journal of Physical Chemistry C 2014, In Press.

16. Farmer, J. A.; Campbell, C. T.; Xu, L.; Henkelman, G., Defect Sites and Their Distributions on $\mathrm{MgO}(100)$ by $\mathrm{Li}$ and $\mathrm{Ca}$ Adsorption Calorimetry. Journal of the American Chemical Society 2009, 131, 3098-3103.

17. Farmer, J. A.; Ruzycki, N.; Zhu, J. F.; Campbell, C. T., Lithium adsorption on MgO(100) and its defects: Charge transfer, structure, and energetics. Physical Review B 2009, 80, 035418. 18. Starr, D. E.; Campbell, C. T., Low-temperature adsorption microcalorimetry: $\mathrm{Pb}$ on MgO(100). Journal of Physical Chemistry B 2001, 105, 3776-3782.

19. Starr, D. E.; Bald, D. J.; Musgrove, J. E.; Ranney, J. T.; Campbell, C. T., Microcalorimetric measurements of the heat of absorption of $\mathrm{Pb}$ on well-defined oxides: $\mathrm{MgO}(100)$ and $\mathrm{p}(2 \mathrm{x} 1)$-oxide on $\mathrm{Mo}(100)$. Journal of Chemical Physics 2001, 114, 3752-3764.

20. Smedh, M.; Diaz, S. F.; Campbell, C. T., Adsorption and adhesion energies of Pb on (1x1)- $\mathrm{Mo}_{2} \mathrm{C} / \mathrm{Mo}(100)$ by calorimetry. Physical Review B 2003, 67, 205401.

21. Farmer, J. A.; Baricuatro, J. H.; Campbell, C. T., Ag Adsorption on Reduced $\mathrm{CeO}_{2}(111)$ Thin Films. Journal of Physical Chemistry C 2010, 114, 17166-17172. 
22. Sharp, J. C.; Yao, Y. X.; Campbell, C. T., Silver Nanoparticles on Fe3O4(111):

Energetics by Ag Adsorption Calorimetry and Structure by Surface Spectroscopies. Journal of Physical Chemistry C 2013, 117, 24932-24936.

23. Starr, D. E.; Diaz, S. F.; Musgrove, J. E.; Ranney, J. T.; Bald, D. J.; Nelen, L.; Ihm, H.; Campbell, C. T., Heat of absorption of $\mathrm{Cu}$ and $\mathrm{Pb}$ on hydroxyl-covered $\mathrm{MgO}(100)$. Surface Science 2002, 515, 13-20.

24. Fischer-Wolfarth, J. H.; Farmer, J. A.; Flores-Camacho, J. M.; Genest, A.; Yudanov, I. V.; Rosch, N.; Campbell, C. T.; Schauermann, S.; Freund, H. J., Particle-size dependent heats of adsorption of $\mathrm{CO}$ on supported Pd nanoparticles as measured with a single-crystal microcalorimeter. Physical Review B 2010, 81, 241416.

25. Bebensee, F.; Zhu, J. F.; Baricuatro, J. H.; Farmer, J. A.; Bai, Y.; Steinruck, H. P.; Campbell, C. T.; Gottfried, J. M., Interface Formation between Calcium and Electron-Irradiated Poly(3-hexylthiophene). Langmuir 2010, 26, 9632-9639.

26. Diaz, S. F.; Zhu, J. F.; Harris, J. J. W.; Goetsch, P.; Merte, L. R.; Campbell, C. T., Heats of adsorption of $\mathrm{Pb}$ on pristine and electron-irradiated poly(methyl methacrylate) by microcalorimetry. Surface Science 2005, 598, 22-34.

27. Etzel, K. D.; Bickel, K. R.; Schuster, R., Heat Effects upon Electrochemical Copper Deposition on Polycrystalline Gold. Chemphyschem 2010, 11, 1416-1424.

28. Bickel, K. R.; Etzel, K. D.; Halka, V.; Schuster, R., Microcalorimetric determination of heat changes caused by overpotential upon electrochemical Ag bulk deposition. Electrochimica Acta 2013, 112, 801-812.

29. Silbaugh, T. L.; Karp, E. M.; Campbell, C. T., Surface kinetics and energetics from single crystal adsorption calorimetry lineshape analysis: Methyl from methyl iodide on Pt(111). Journal of Catalysis 2013, 308, 114-121.

30. Ajo, H. M.; Ihm, H.; Moilanen, D. E.; Campbell, C. T., Calorimeter for adsorption energies of larger molecules on single crystal surfaces. Review of Scientific Instruments 2004, 75, 4471-4480.

31. Etzel, K. D.; Bickel, K. R.; Schuster, R., A microcalorimeter for measuring heat effects of electrochemical reactions with submonolayer conversions. Review of Scientific Instruments $2010,81,034101$.

32. Stuckless, J. T.; Frei, N. A.; Campbell, C. T., A novel single-crystal adsorption calorimeter and additions for determining metal adsorption and adhesion energies. Review of Scientific Instruments 1998, 69, 2427-2438.

33. Stuckless, J. T.; Frei, N. A.; Campbell, C. T., Pyroelectric detector for single-crystal adsorption microcalorimetry: analysis of pulse shape and intensity. Sensors and Actuators BChemical 2000, 62, 13-22.

34. King, D. A.; Wells, M. G., Molecular-beam investigation of adsorption kinetics on bulk metal targets - nitrogen on tungsten. Surface Science 1972, 29, 454.

35. Deng, R. P.; Herceg, E.; Trenary, M., Characterization of methylidyne on Pt(111) with infrared spectroscopy. Surface Science 2004, 573, 310-319.

36. Lew, W.; Lytken, O.; Farmer, J. A.; Crowe, M. C.; Campbell, C. T., Improved pyroelectric detectors for single crystal adsorption calorimetry from 100 to $350 \mathrm{~K}$. Review of Scientific Instruments 2010, 81, 024102. 


\section{Figures}

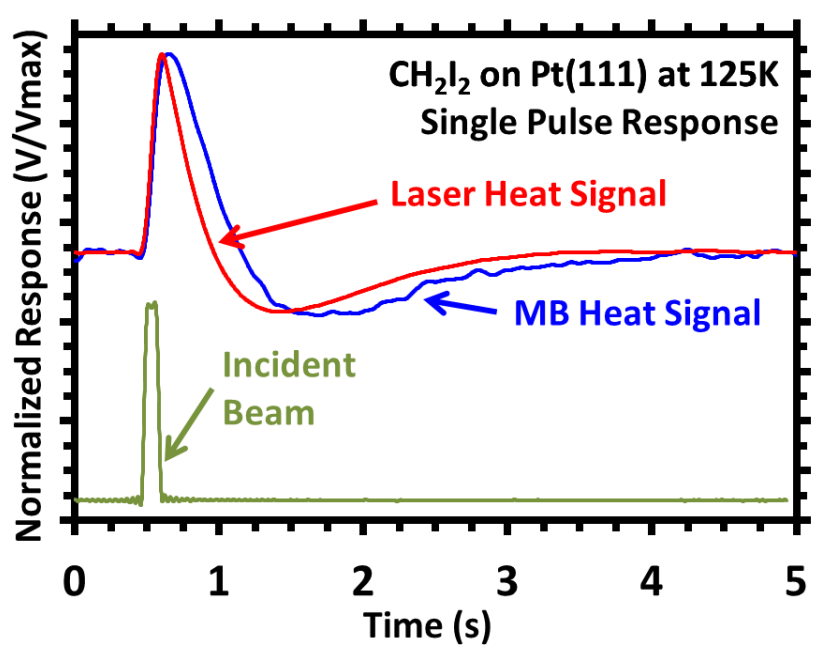

Figure 1. Comparison of typical normalized response signals measured for single pulses of the laser beam and the molecular beam (MB), for a case where the latter shows peak broadening due to slow kinetics. Also shown is the incident beam profile, which is approximately a square wave. This profile is essentially identical for both the laser and the molecular beams, and its shape is determined by the diameter of the beam, the geometry of the chopper wheel and its rotational velocity. The incident beam profile shown here was collected by measuring the direct molecular beam intensity with a quadruple mass spectrometer (QMS). 


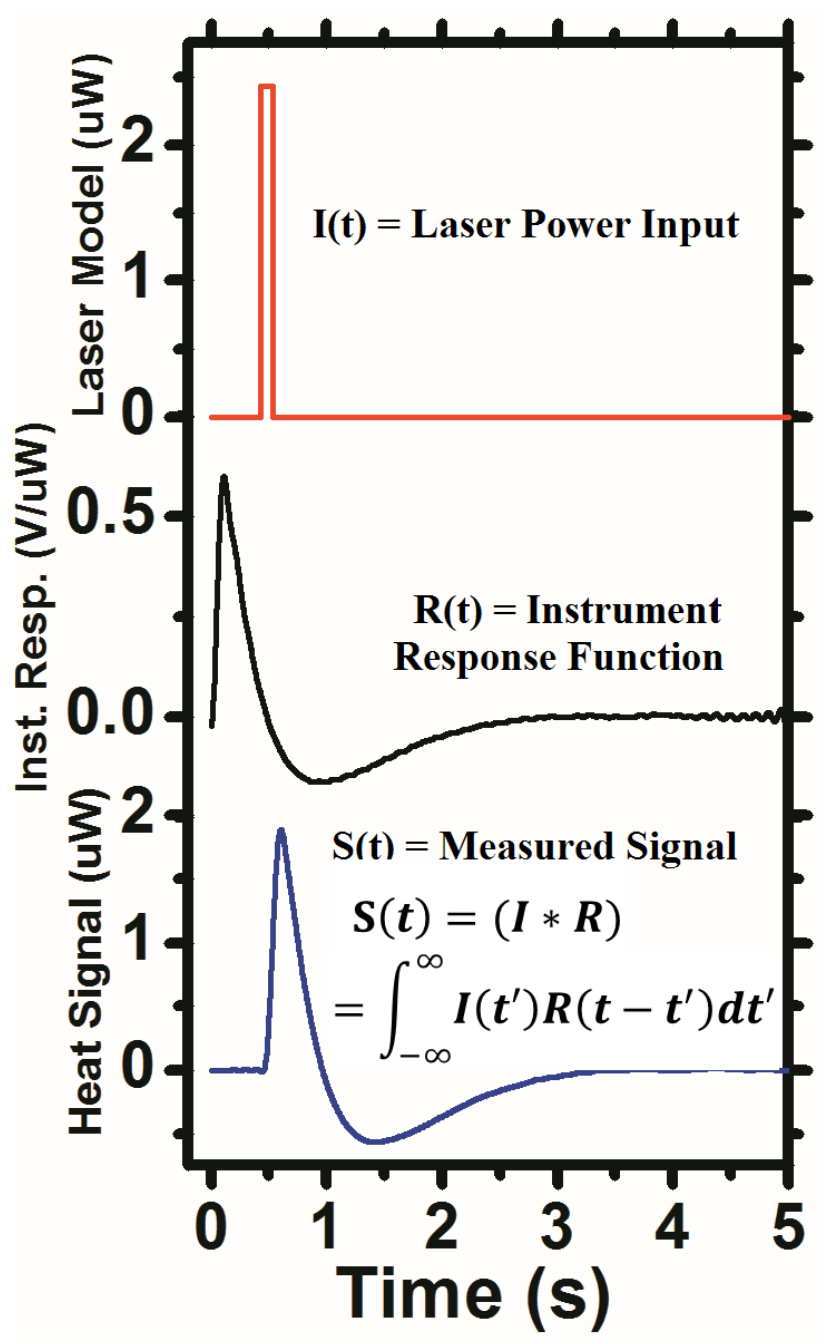

Figure 2. A typical heat deposition profile (I(t), here a square-wave model of the incident laser power) is convoluted with the instrument response function $(\mathrm{R}(\mathrm{t}))$ to yield the measured detector signal $(\mathrm{S}(\mathrm{t}))$. Here, $\mathrm{R}(\mathrm{t})$ was actually calculated by deconvolution of the known $\mathrm{I}(\mathrm{t})$ from the measured $\mathrm{S}(\mathrm{t})$, as detailed in the text. Because the instrument response is partially determined by the quality of the physical contact between the heat detector and the sample, it is necessary to extract the instrument response function every time a new contact is established (once for each heat-versus-coverage run). The laser power input function is modelled here as a square wave. It was also modelled as a trapezoidal wave based on the known open/close profile of the chopper in this system, which produced an identical instrument response function (see text). 


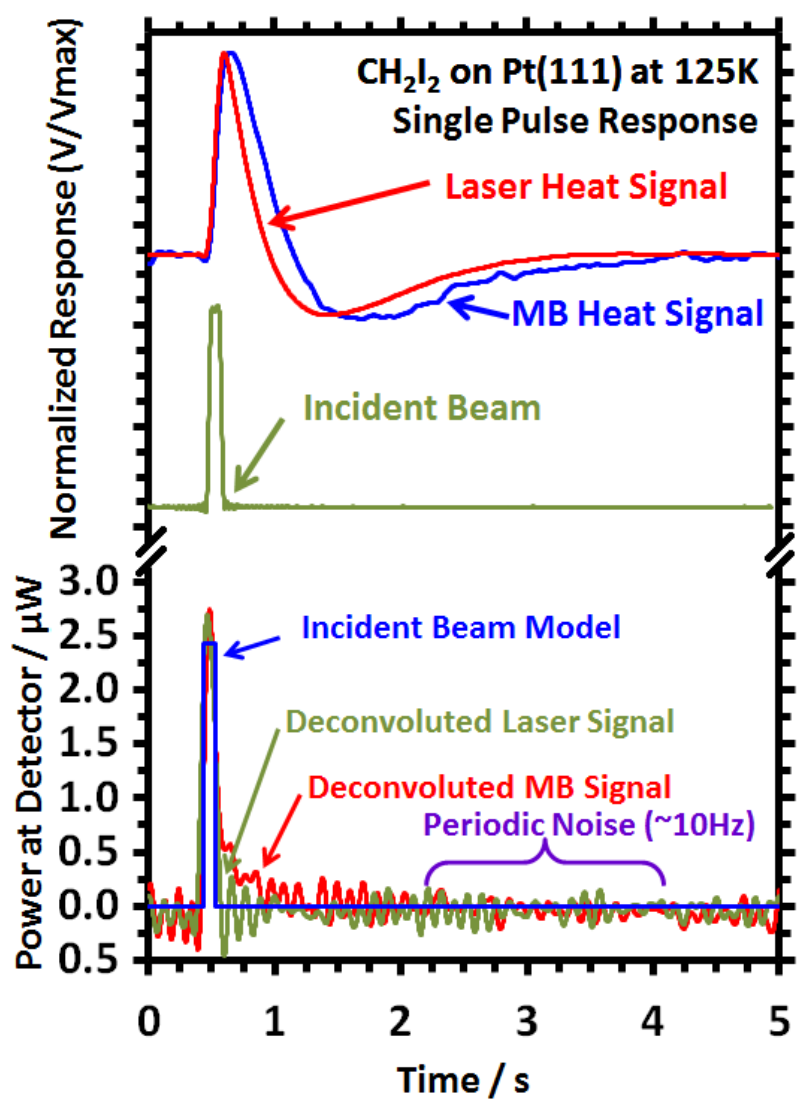

Figure 3. Top: The incident beam profile and typical response signals measured for the laser beam and the molecular beam (MB), reproduced from Fig. 1. Bottom: the incident power at the detector from the molecular beam as a function of time, extracted by deconvoluting the instrument response function from the MB heat signal using the FFT method. The square wave incident beam model is also shown. The deconvoluted MB signal is the result of deconvoluting the MB heat signal with the instrument response function, while the deconvoluted laser signal is the result of deconvoluting a single laser heat signal with that same instrument response function. (The average of many laser heat signals is used to extract the instrument response function.) It can be seen that for this individual MB pulse, the deconvoluted heat signal is broader than the incident beam, showing that heat is deposited on a slower time scale than during the laser pulse. The deconvoluted pulse tail decays quickly towards the baseline zero, but continues to be distinguishable above the baseline noise for almost $1 \mathrm{~s}$ before being overwhelmed by the periodic noise introduced by this method. 


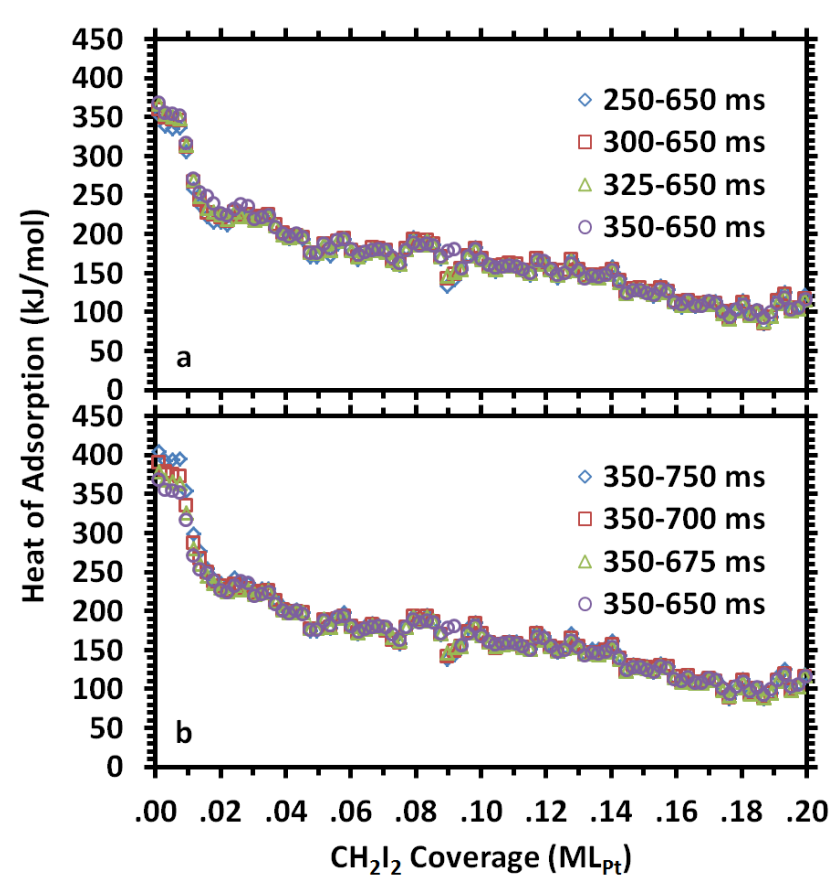

Figure 4. Differential heat of adsorption of $\mathrm{CH}_{2} \mathrm{I}_{2}$ on clean $\mathrm{Pt}(111)$ at $125 \mathrm{~K}$ as a function of $\mathrm{CH}_{2} \mathrm{I}_{2}$ coverage, extracted by integrating each deconvoluted pulse (see Fig. 3) over the different integration windows stated. By increasing the integration window by time increments that are $1 / 4$ of the period of the dominant periodic noise by moving either to earlier start times (a) or later stop times (b), this shows that no systematic change in heat is found at most coverage. Thus, the periodic noise does not have a significant impact on the choice of window for integration.

However, at very low coverages, which had the most broadened heat signal pulses, increasing the integration window to longer times does increase the heats systematically as it captures more and more of the slow heat deposition. The heat stops increasing when this is extended to $\sim 1000$ ms, after which the effect saturates (see Fig. 5). 


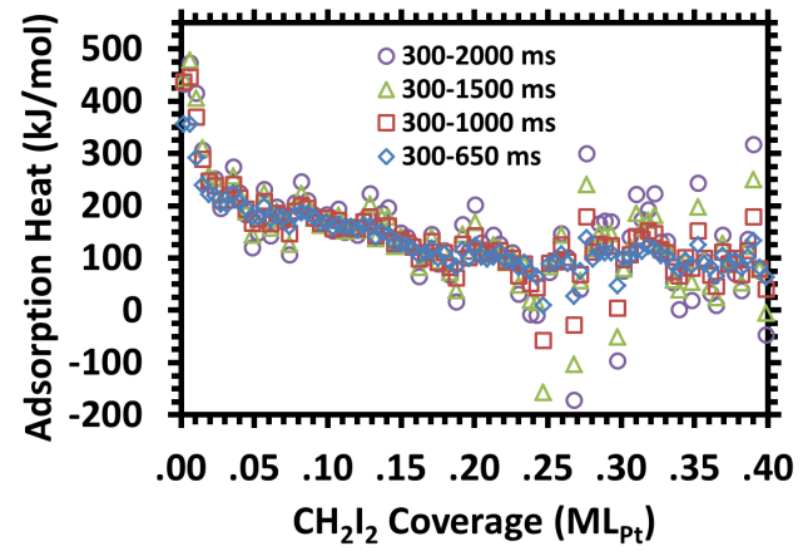

Figure 5. Differential heat of adsorption of $\mathrm{CH}_{2} \mathrm{I}_{2}$ on $\mathrm{Pt}(111)$ at $125 \mathrm{~K}$ as a function of $\mathrm{CH}_{2} \mathrm{I}_{2}$ coverage obtained using different time window widths for integrating the heat pulses. These heats were extracted by integrating each deconvoluted pulse (see Fig. 3) over the specific time windows shown. A large enough window must be selected to capture all of the heat deposited (see low coverage results in Fig. 4), and ideally one would integrate across the full time window. This shows, however, that as the integration window gets larger, the noise increases, although the average heat remains unchanged at most coverages. At low coverages where the heat signal is most broadened, the heat increase with integration window until 300-1000 ms, above which it has the same average. This shows that whatever slow kinetics are occurring on the surface, they have already completed by $1000 \mathrm{~ms}$. 


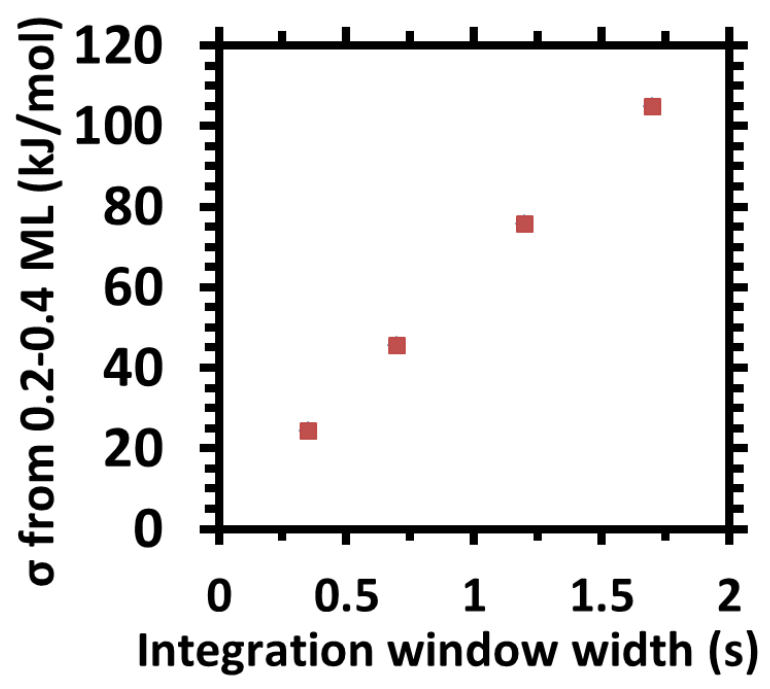

Figure 6. The standard deviation of the heat measured in Fig. 5 over the coverage range from 0.2 to $0.4 \mathrm{ML}$ (where the heat is nearly constant with coverage) as a function of integration window width beginning at $0.3 \mathrm{~s}$. The standard deviation increases proportional to the integration window width. The best selection of window width was decided based on seeking to minimize this noise while simultaneously capturing all of the heat deposition occurring for a pulse of gas (which time duration varies depending upon the reaction being studied and surface temperature). 


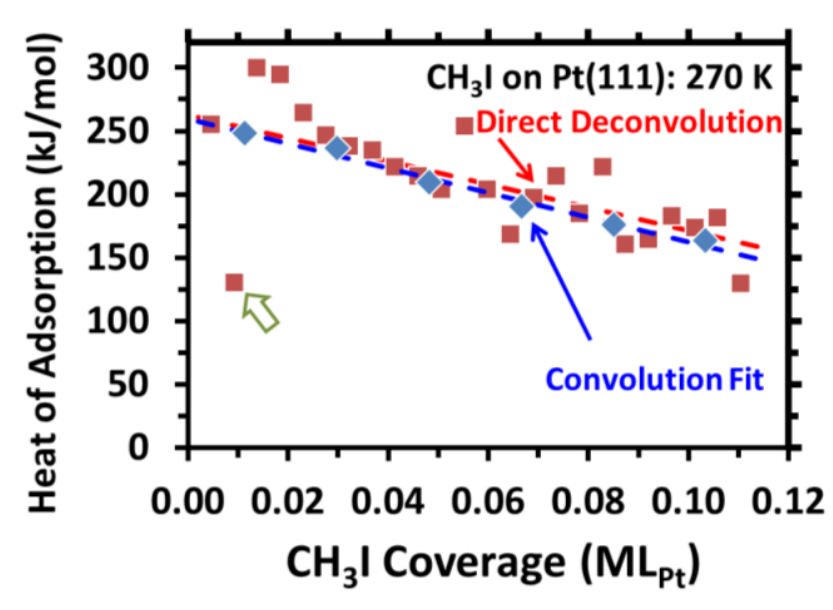

Figure 7. Comparison of differential heat of adsorption versus coverage results for $\mathrm{CH}_{3} \mathrm{I}$ on clean $\mathrm{Pt}(111)$ at $270 \mathrm{~K}$, between the convolution fitting method of Silbaugh et al. ${ }^{29}$ (blue points) and the direct deconvolution method of this paper (red points). In Silbaugh's method, four pulses were averaged to reduce noise. Each of the points of Silbaugh is essentially the average of the four adjacent points from the method of this paper. The best-fit lines shown are very similar, and represent the equations: $\mathrm{y}=(259-969 \mathrm{x}) \mathrm{kJ} / \mathrm{mol}$ using the convolution fit method, and $\mathrm{y}=(263-$ $911 \mathrm{x}) \mathrm{kJ} / \mathrm{mol}$ using the direct deconvolution method. 


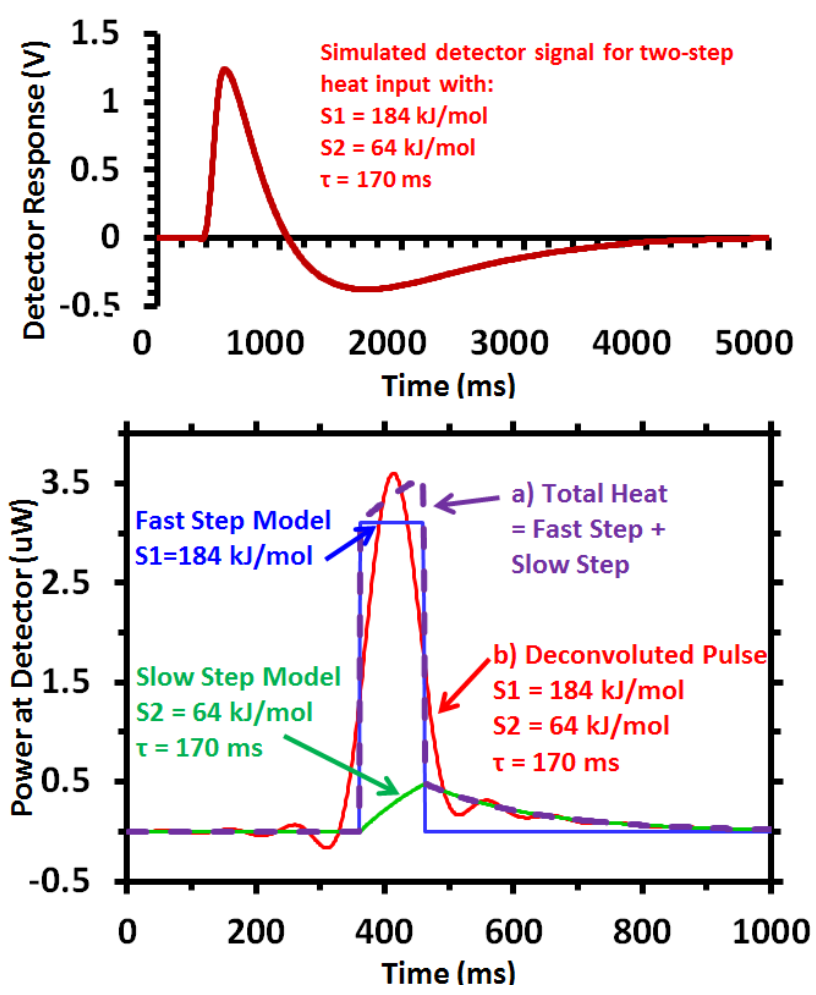

Figure 8. Bottom: Comparison of the input power versus time due to the heat of adsorption followed by dissociation for $\mathrm{CH}_{3} \mathrm{I}$ adsorption on $\mathrm{Pt}(111)$ at $270 \mathrm{~K}$ for a typical $0.0046 \mathrm{ML}$ pulse within the coverage range $0-0.02 \mathrm{ML}$, as calculated from:

(a) The two-step model of Silbaugh et al. ${ }^{29}$ for this condition wherein $\mathrm{CH}_{3} \mathrm{I}$ first adsorbs molecularly with a heat of $184 \mathrm{~kJ} / \mathrm{mol}$ and then dissociates via a first-order reaction with time constant $\tau=170 \mathrm{~ms}$ and heat of $64 \mathrm{~kJ} / \mathrm{mol}$ (purple, dashed curve). Also shown are the separate heat inputs from the fast step (blue) and slow step (green). The curve at the top shows the simulated signal waveform for this same exact two-step heat input model, as generated using the simulation method of Silbaugh et al. which sums heat-scaled waveforms for its two steps, using the measured lineshape for the signal response to laser heating for the first, fast step and its convolution with first-order exponential decay kinetics for the slow step.

(b) Deconvolution of top simulated signal to remove the instrument response function (shown in Fig. 2).

Curves (a) and (b) agree, except for the damped periodic noise that results from deconvoluting over the finite time window used (4000 points over $5 \mathrm{~s}$ ). 


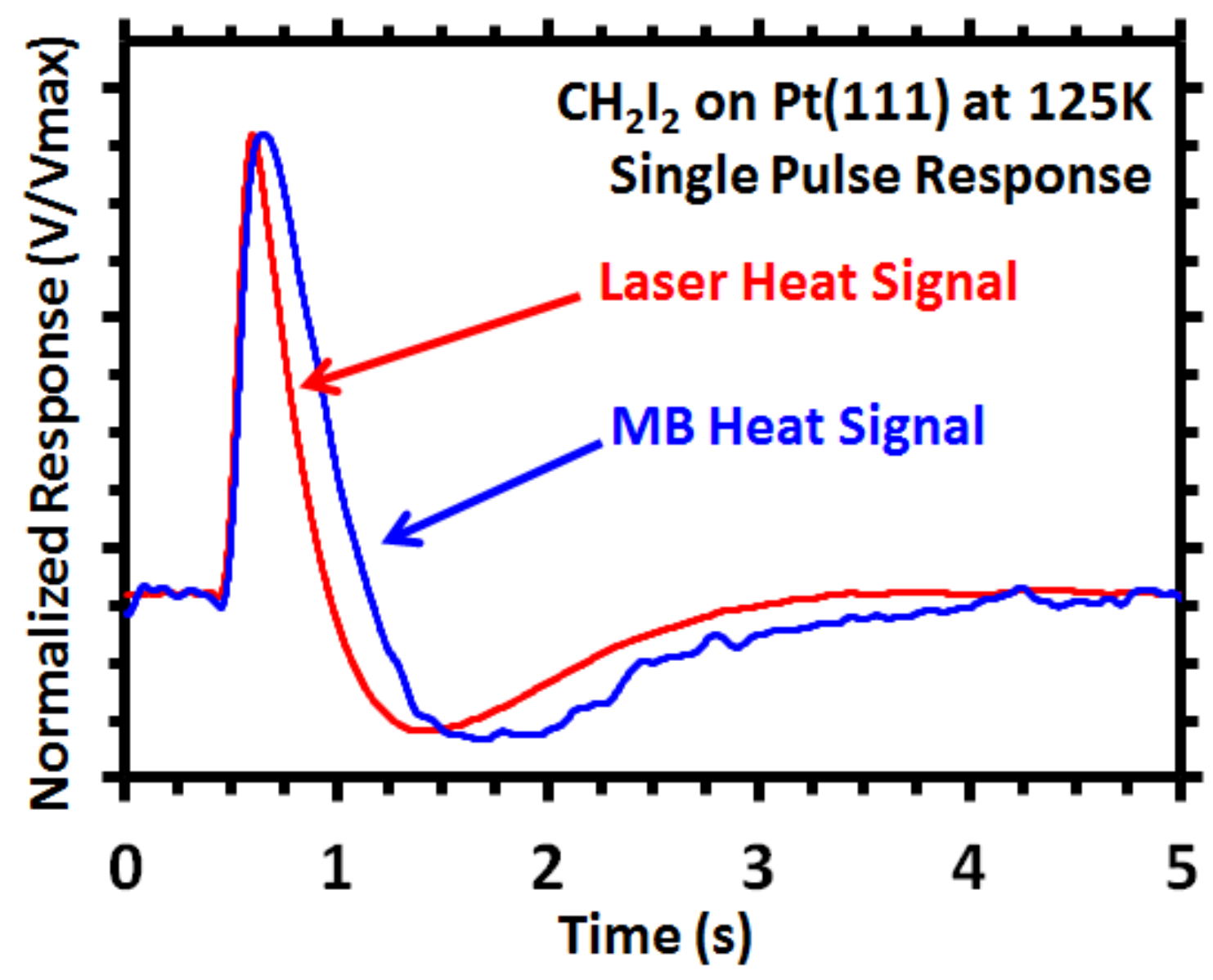

Graphical abstract 
Method for Direct Deconvolution of Heat Signals in Transient Adsorption Calorimetry,

by Christopher A. Wolcott and Charles T. Campbell, 